\title{
LA INCLUSIÓN SOCIAL CON OPCIONES PRODUCTIVAS
}

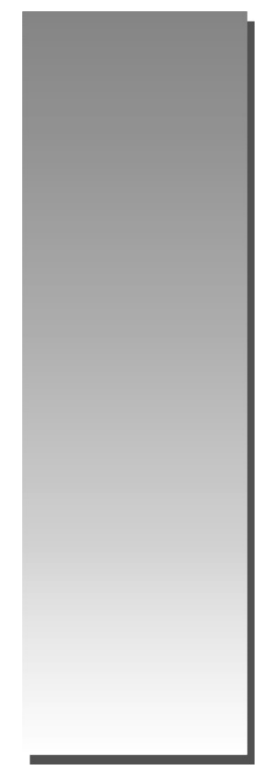

Enrique Huaco Pastor*

enrique_huaco@yahoo.com

\section{RESUMEN}

La inclusión social es un concepto que en un ámbito general implica el proceso de incorporar social, económica, política y culturalmente a la comunidad nacional a los grupos sociales excluidos y vulnerables. En un ámbito operativo abarca la noción de pobreza y de desigualdades sociales en una colectividad, pero no se agota en ellas. Mientras que pobreza y las desigualdades sociales constituyen una categoría económica que alude a los ingresos de las personas, el concepto de inclusión social incluye otras dimensiones; entre ellas, la educación, la salud, la incorporación al mercado, la equidad en las oportunidades que implican el bienestar y el desarrollo integral de los integrantes de una colectividad.

Podemos reconocer que la categoría de la pobreza trae consigo la categoría de las desigualdades sociales, falta de integración de los individuos con la vida y los recursos institucionales de su medio. Así mismo, se evidencia la brecha que existe entre el medio urbano con el medio rural cuya separación parece dibujar los matices de la exclusión entre grupos sociales que conviven dentro de un mismo territorio pero que, sin embargo, las diferencias conllevan a la ruptura de los vínculos socioculturales e históricos que los unían.

Nuestro planteamiento corresponde a la formulación de propuestas prácticas relacionadas con la reforma y modernización de las instituciones públicas del gobierno central, regional y local y entre ellas las universidades con competencias para la promoción, organización y asistencia técnica de empresas de economía solidaria, diseñadas en base al establecimiento de cadenas productivas y de formación clúster como una modalidad de asociatividad y de articulación empresarial, en la modalidad de redes empresariales.

Palabras clave: Inclusión social, exclusión social, economía solidaria, formas asociativas de empresas

* Licenciatura en Administración, Magíster en Administración, Docente Asociado de la Facultad de Ciencias Administrativas de la Universidad Nacional Mayor de San Marcos 


\begin{abstract}
Social inclusion is a concept that in a general area involves the process of incorporating social, economically, politically and culturally to the national community social groups excluded and vulnerable, in an operational area covers the notion of poverty and social inequality in a community, but is not limited to them. While poverty and social inequalities are an economic category that refers to the income of people, the concept of social inclusion includes other dimensions, including education, health, market entry, equity in opportunities involving welfare and development of the members of a community. We recognize that poverty category category brings social inequalities lack of integration of individuals with life and institutional resources of their environment. So it is evident the gap between urban to rural areas whose separation seems to draw the nuances of social exclusion among groups living within the same territory but nevertheless, differences lead to the breakdown of cultural ties and historical that bound.

Our approach corresponds to the formulation of practical proposals relating to the reform and modernization of public institutions of central government, regional and local universities including powers for the promotion, organization and support of solidarity economy enterprises, designed based the establishment of production chains and cluster formation as a form of partnership and joint enterprise, in the form of enterprise networks.
\end{abstract}

Keywords: Social inclusion, social exclusion, economic solidarity, associative forms of businesses

\section{INTRODUCCIÓN}

En los últimos 25 años, en el Perú viene creciendo el PBI constantemente a un promedio del $7 \%$, crecimiento que viene generando un mejor nivel y calidad de vida, pero también incrementa las brechas sociales, expresadas en las desigualdades económicas y sociales, habiendo registrado un escenario aun de exclusión social, ocasionado entre otros factores por la desprotección del Estado, principalmente en los sectores de la sierra y selva del Perú.

La exclusión social involucra el fenómeno de la vulnerabilidad social que se evidencia en la incapacidad de las personas o de un lugar geográfico para aprovechar las oportunidades, disponibles en distintos ámbitos socioeconómicos, para mejorar su situación de bienestar o impedir su deterioro

Esta vulnerabilidad se genera por tres causas principalmente, las cuales son la precariedad e inestabilidad laboral, las crisis de la familia y conflictos en las comunidades que se manifiesta por la ruptura del vínculo social dentro de los colectivos sociales, y no solamente por una cuestión de pobreza y desigualdad económica. Es relievar que uno de los problemas que más afecta a la sociedad es la enorme cantidad de personas que carecen de condiciones dignas de vida, de tener una alimentación adecuada, una vivienda digna, una vestimenta en condiciones saludables, educación y salud, etc. en otras palabras, de satisfacer sus necesidades básicas; para ello es un monto mínimo de ingresos Toda persona sin conocimientos es vulnerable y es proclive a que se vuelva miserable, no puede desenvolverse en la sociedad y se convierte en una persona sujeta al apoyo caritativo del Estado.

Podemos reconocer que las causas de la exclusión social son multidimensionales que se enmarcan en un proceso de pérdida de integración o participación del individuo en la sociedad,en uno o varios de estos ámbitos:

- Económico (en las áreas de la producción o en el consumo).

- Político-legal (participación política, sistema administrativo, protección social, etc.)

Desde el siglo pasado, todos los gobernantes han dicho "Hay que enseñarles a los pobres a pescar y no regalarle el pescado", después crean los programas sociales asistencialistas, que no pueden, no saben las reglas de juego que les permita cumplir con su palabra.

Los campesinos de la sierra, los pescadores de las caletas o los nativos de la selva no se alimentan del conocimiento por falta de oportunidades. Cuando no se coordinan correctamente los activos y el aprovechamiento de las estructuras de oportunidades, se puede apreciar claramente un aumento de la precariedad de los hogares, y cuando esto se convierte en un problema grave, conduce casi con seguridad a la marginalidad y a la exclusión social.

Frente a esta situación, el gobierno peruano, en el 2011, ha adoptado y viene desarrollando políticas de inclusión social, implementando principalmente un conjunto de programas so- 
ciales asistencialistas orientadas a promover la participación social y mejorar su nivel de vida. Así también ha formulado políticas de carácter productivo, tales como de promover y ejecutar inversiones en infraestructura vial, electrificación, agua, de banda ancha de fibra óptica, que en la parte del desarrollo del estudio, procederemos a su especificación.

\section{MÉTODOS}

La investigación será de tipo descriptivoexplicativo por sus características. La metodología de investigación que se empleará será lograr la demostración y contrastación de las hipótesis correspondiendo a la metodología descriptivaexplicativa. La investigación se concentrará en la obtención de un escenario social, económico y cultural representativo para el sector urbano y otro escenario para el sector rural, describiendo sus principales características y extrayendo propuestas con contenido productivo que contribuya como aportes a las políticas de inclusión social.

El escenario social, económico y cultural del Perú presenta muy poca presencia de los gobiernos en materia de inclusión social con opciones productivas, siendo de atención con énfasis en políticas sociales asistencialistas, que por prospectiva exige que para hacer frente a los desafíos de la globalización, competividad e innovación , está haciendo que surga la necesidad de realizar propuestas productivas en el marco de una economía de mercado con características de empresas en el marco de una economía solidaria y ella dentro de la economia de mercado y promoviendo las sinergias.

\section{RESULTADOS}

Nuestro planteamiento corresponde a la formulación de propuestas prácticas relacionadas con la reforma y modernización de las instituciones públicas del gobierno central, regional y local, y entre ellas las universidades con competencias para la promoción, organización y asistencia técnica y financiera de empresas de economía solidaria, diseñadas en base al establecimiento de cadenas productivas y de formación de conglomerados y clústers como una modalidad de asociatividad y de articulación empresarial, en la modalidad de redes empresariales.

Enfrentar con posibilidades de éxito se requiere el soporte de las universidades y centros tecnológicos a los desafíos que planteen los vertiginosos avances en las ciencias y tecnologías, así como en las disciplinas emergentes y de la innovación que se viene impulsando con intensidad en el mundo, así como los requerimientos que demanda el desarrollo nacional, con crecimiento económico con inclusión social, en constituirnos en competitivos exportadores de bienes y servicios con la generación de valor agregado, que impulse unidades empresariales dentro de un marco y articulación de conglomerados empresariales, conformación de cadenas productivas y clúster, que presentemos una mayor apertura y cobertura a la integración en redes con establecimientos universitarios nacionales y extranjeras, así como el concurrir en apoyo y soporte del gobierno nacional, regional, local y central, demanda que la propuesta de estructura organizativa de la UNMSM, tendría que asumir progresivamente un nuevo modelo de organización más flexible, horizontal , estructurado por procesos claves, conformando módulos con el contenido de procesos, desconcentrando sus estructuras administrativas en unidades de servicios especializados en las que estarán incluidas una buena parte de las funciones de apoyo: así como descentralizando administrativamente las funciones de asesoramiento que se muestran el estructura orgánica actual de la UNMSM, pero captando el capital humano y dinamizándolo en la prestación de servicios a las organizaciones de la sociedad.

\section{DISCUSIÓN}

\section{Escenario de la exclusión e inclusión social}

Para abordar la investigación de la inclusión Social, es conveniente conceptualizar lo que implica el concepto, así mismo, situarlo en el marco de la perspectiva predominante entre los países emergentes, entre ellos, el Perú. La Inclusión social como escenario comprende un proceso de acercamiento progresivo de una situación de desintegración social, cuyos mecanismos estructurales impiden a ciertos grupos sociales participar plenamente en las dimensiones económica, social, política y cultural de la sociedad. Frente a la ruptura del vínculo social dentro de los colectivos sociales se da la exclusión social y no solamente por una cuestión de pobreza y de desigualdad económica sino también debido a la falta de participación social, por tanto, el concepto del estatus de esa población deriva en la carencia e incapacidad en ejercitarse como ciudadanos plenos. 
El impacto de la exclusión social no sólo radica en el nivel de vida y/o calidad de vida de las personas que pertenecen a una sociedad que las margina, también existe un efecto psicológico; estas personas sienten que no pertenecen a la sociedad y que incluso es ella misma la que no les permite ingresar y/o no le interesa incluirlos; este estado negativo actúa a manera de murallas las cuales se cimientan y terminan separando definitivamente al individuo de su grupo social originario

La vulnerabilidad social es: "La incapacidad de una persona o de un lugar para aprovechar las oportunidades, disponibles en distintos ámbitos socioeconómicos, para mejorar su situación de bienestar o impedir su deterioro". El aumento de la precariedad laboral incidió, en los últimos tiempos, en que muchas familias no lleguen a tener un monto mínimo de dinero, lo cual deriva en un aumento de la vulnerabilidad en los hogares.

El proceso de la inclusión social demandara un esfuerzo notable del Estado, mediante políticas públicas en varios sectores económicos y sociales, pero también de las empresas privadas, comprometiéndose en dar mayor énfasis en la responsabilidad social y también de responsabilidad ambiental.

Dichas políticas públicas deberán involucrar no solamente políticas públicas mediante programas sociales, sino con políticas de desarrollo y crecimiento de inversión en la infraestructura física , principalmente en carreteras que acerquen a los sectores rurales al mercado de las zonas urbanas, ampliar la infraestructura con energía eléctrica y autopistas tecnológicas con inversiones en bandas de fibra óptica, para que la población rural que acusa los mayores niveles de pobreza y de desigualdad puedan ser integrados al mercado. $\mathrm{Al}$ respecto, el Estado plantea el Programa de Electrificación Rural: electricidad para $738 \mathrm{mil}$ viviendas al año 2016 beneficiando a 3,36 millones de personas, mientras el Fondo de Inversiones en Telecomunicaciones tiene previsto llegar a todos los distritos aislados con telefonía fija y celular.

Los programas de alivio de la pobreza, los del acceso a las oportunidades y las estrategias sostenidas de generación de ingresos, seguridad alimentaria y empleo productivo serán la esencia de la inclusión social.

El compromiso de cumplimiento de los programas sociales es priorizar el trabajo en las zonas rurales, de donde de cada seis peruanos de diez peruanos son pobres y uno de cada 4 pasa hambre.

La vulnerabilidad se genera por tres causas principalmente, las cuales son precariedad e inestabilidad laboral, la desprotección del Estado por su achicamiento y a las crisis de la familia y la comunidad. Esta vulnerabilidad se expresa en la enorme cantidad de personas que carecen de condiciones dignas de vida, de tener una alimentación adecuada, una vivienda digna, una vestimenta en condiciones, falta de educación, etc. En otras palabras, de satisfacer sus necesidades básicas; para ello es necesario un monto mínimo de ingresos.

\section{Programas sociales asistencialistas del gobierno}

El Estado peruano proyecta reducir la pobreza al $15 \%$ al año 2018 , y se señala como avances de la gestión al año 2012 el lanzamiento de los siguientes programas sociales asistenciales.

Cuna Más atiende a 1700 menores de 2 a 3 años del ámbito rural y para el año 2016, de las madres que trabajan se espera dar atención integral a 260 mil niños. El programa contará con la atención de profesoras de educación inicial para el cuidado de los niños y entregará pañales, leche y alimentación de manera gratuita. Para el año 2012, el programa Cuna Más contará con una inversión de 191 millones de soles para cubrir la meta de atención a 54 mil niños de los 455 distritos más pobres del país que ya son atendidos por el programa Juntos. La meta es llegar a los 800 distritos más pobres del país.

Beca 18 es una estrategia del Gobierno para facilitar el acceso de estos jóvenes talentosos a la educación superior. Son cinco mil becas para este 2012. Ya se ha iniciado el proceso para la primera convocatoria ordinaria. Esta incluye a jóvenes de provincias y distritos pobres del país, que les permitirá ingresar a una universidad o instituto a carreras que se han definido como elegibles Es una beca integral. Cubre, por una parte, el servicio educativo de la universidad que se haya seleccionado, movilidad local, materiales, y transporte si estudia fuera de esa provincia. Están incluyendo un seguro de salud, de vida y un programa de tutoría, porque se ha probado que muchos de los futuros becarios son la primera generación de su familia que va dirigida a todos los jóvenes de distritos de pobreza que han terminado en un colegio público y que vienen de familias con necesidades económicas. Y la especial que es una 
beca más focalizada, orientada a ciertos grupos que han sido excluidos, como poblaciones indígenas, afro peruanas, licenciados de las FF.AA., jóvenes de zona de emergencia o de frontera, con necesidades especiales o discapacidad. Esta beca incluye un proceso de nivelación previa, y será para el segundo semestre del año 2012.

Pensión 65: beneficiará a 173 mil adultos mayores en 990 distritos a finales del 2012, llegar a 247 personas. El titular del Ministerio de Economía y Finanza indicó que el programa social Pensión 65 tendrá una partida de apertura de 241 millones de soles el próximo año, con lo cual se busca atender a unos 167 mil adultos mayores.

El monto destinado irá sobre todo a zonas de extrema pobreza y se extenderá a 1.076 distritos de todo el país, el presupuesto de 822 millones de nuevos soles para atender a la población de 14 departamentos donde la pobreza monetaria es de $50 \%$. Asimismo, anunció que el citado programa social alcanzará en el 2014 una cobertura de 1.777 distritos que tienen más del $50 \%$ de pobreza a nivel nacional.

Jóvenes a la Obra es el programa nacional de empleo juvenil, resultado de la iniciativa para trabajar por los jóvenes de 15 a 29 años, sobre todo de aquellos que están desempleados, en situación de pobreza y excluidos. Este programa no solo busca capacitarlos sino también insertarlos al mercado y generar emprendimiento para el desarrollo de un trabajo digno e independiente.

El programa Samu plantea conformar un sistema de atención médica móvil de urgencia, es un servicio médico integral que permitirá a todas las personas, especialmente las de menos recursos económicos, recibir asistencia de manera oportuna y con calidad, cuando se presente una urgencia o emergencia y en el lugar donde se encuentre de manera rápida, eficiente y gratuita.

Programa de Apoyo directo a los más Pobres (Juntos), en el año del 2012, fue ampliado y brinda atención a 495 mil hogares de 14 regiones, al final del año 2012 atenderá 722 hogares.

Fondo de Cooperación para el Desarrollo (Foncodes) es un programa que se orienta a buscar la rehabilitación y dar mantenimiento a la infraestructura de riego e infraestructura vial existente y generar empleo temporal en las áreas rurales pobres de la sierra y selva. Los proyectos que se ejecutan en asociación con la población organizada y los gobiernos locales comprenden obras de refacción y recuperación de canales de riego, bocatomas, reservorios, caminos, puentes peatonales y trochas carrozables, entre otros. Se trata de un programa de intervención temporal que busca estimular las economías locales para mitigar los posibles impactos de la crisis económica internacional.

El Programa de Apoyo Crediticio a la Pequeña y Microempresa (PAME) tiene como objetivo general contribuir al desarrollo y consolidación del mercado de los servicios financieros de créditos para los pequeños y microempresarios de las zonas rurales y urbano-marginales del país, que no tenían acceso al sistema financiero. La población beneficiaria son los microempresarios de las áreas urbano-marginales que realizan actividades productivas en los sectores comercio, servicios y manufactura, así como productores rurales de café, cacao, papa, granadilla, flores, entre otros, principalmente asociados en cadenas productivas.

Programa Qali warma: brinda servicios de desayuno y almuerzos a escuelas públicas, se atenderá a 3,9 millones de infantes. Programa de Redes Rurales beneficiará a 1,200 escuelas con infraestructura, dotación de docentes, conectividad con internet, formación de docentes bilinguies, etc. Programa de Tambos beneficiará a poblaciones alejadas y garantizará la presencia del Estado en salud, educación, seguridad, justicia y comunicaciones. Al mes de setiembre de 2010, están en construcción 190 tambos que beneficiarán a 156 familias.

Programa de Sierra Exportadora es un servicio dirigido a productores y empresarios de las zonas andinas del país que requieren apoyo para formar asociaciones de productores que les permita alcanzar objetivos comunes tales como alcanzar un volumen de producción de un mismo producto con marca colectiva para abastecer una demanda de un mercado específico o el acceso a un financiamiento que requiere garantías que sean cubiertas proporcionalmente por parte de cada uno de los participantes. También pueden asociarse para la adquisición de un volumen de materia prima, o desarrollar tecnologías para el beneficio común. Este es uno de los principales programas de desarrollo productivo, cuya finalidad es difundir en los andes la producción del arándano, frambuesa, cereza, fresa, mora y aguay manto, papas entre otros, para la exportación. 
Con respecto a los programas destinados a mejorar la infraestructura básica y de servicios, el programa de construcción y equipamiento de centros educativos es el más conocido, con el $64.9 \%$. El abastecimiento de servicios de agua, desaguie y luz es por el $61.7 \%$, la construcción de caminos y puentes es por el $58.7 \%$ y la construcción y equipamiento de centros de salud por el $53.6 \%$, con programas de infraestructura de sistemas de riego (44.3\%).

Los investigadores de la Maestría de la Universidad de Lima señalan que los programas sociales no reducen la pobreza. Eso es asistencialismo Solo cuando haya una inversión privada de 3,5 millones de dólares, así como trabajo decente para los jefes de hogar se podrá reducir la pobreza.

En los últimos años, el crecimiento económico ha contribuido a reducir la pobreza en más de 28 puntos porcentuales hasta alcanzar una tasa del $30 \%$ hacia el año 2011 , no obstante hay diferencias entre las regiones. También podemos decir que el crecimiento en un $7 \%$ del PBI, aproximadamente los últimos diez años ha sido insuficiente para reducir las brechas, especialmente con el sector rural.

El presupuesto nacional para el año 2013, ascendente a 108, 418'909,559 nuevos soles y tiene un crecimiento del $13,49 \%$ con respecto al presupuesto del año fiscal 2012. También contempla un significativo aumento del $39 \%$ con respecto del presupuesto actual para programas sociales.

En líneas generales, la inversión pública crecerá para el año 2013 en 16\%, el presupuesto de inversión para agua y saneamiento, en $25 \%$; mientras que para los sectores de salud y educación aumentará en más del $10 \%$, evidenciando que con la solidez de la economía se está atendiendo la preocupación del Ejecutivo por impulsar la descentralización, reforma del Estado y su modernización e incrementar el apoyo a programas sociales, así como inversiones productivas. No obstante, estas políticas públicas aún no se extienden a los lugares más apartados del país, en el ámbito rural sin protección del Estado. Por otro lado, en la prestación de los servicios sociales se vienen registrando filtración con indicios de corrupción; asimismo, las instituciones públicas aún mantienen estructuras organizativas obsoletas, puesto que todavía no ha iniciado una reforma y modernización de las organizaciones de la administración pública.

\section{CONCLUSIONES}

1. Dichos planteamientos o propuestas tienen el propósito de promover y fortalecer la reforma y modernización de las instituciones públicas, que mediante la reingeniería de procesos va diseñar renovadas estructuras organizativas formales, que van a gravitar sustantiva y positivamente en los procesos de inclusión social y que lo expresamos en las siguientes acciones:

a. Diseñar e implantar un proceso de descentralización del Ministerio de Producción, transfiriendo las competencias relacionadas con la Dirección de Micro Empresas y Cooperativas a una institución pública descentralizada y que este proceso se extienda en una desconcentración de competencias a los gobiernos regionales y locales. Podría adoptar la denominación de Instituto Nacional de Economía Solidaria (INES), adscrita al Ministerio de Producción. Esta propuesta se sostiene en que los ministerios y sus dependencias internas generalmente asumen competencias normativas o técnicas normativas.

b. Que el INES convoque y concrete alianzas estratégicas con universidades, para que estos centros superiores brinden asistencia técnica en todas las áreas funcionales de las microempresas, pequeñas empresas y cooperativas, así también que estas participen en los distintos centros de innovación y tecnología. En estas alianzas también estarían involucradas las instituciones financieras del Estado.

c. En el sector rural actuar en la promoción y constitución de cooperativas agrarias conformada por los campesinos y comunidades campesinas, esta acción implica dinamizar Foncodes estableciendo metas de un mayor número de titulaciones de los predios agrícolas, así también el establecimiento de cadenas productivas y mediante la asociatividad conformar clúster y conglomerados empresariales. Asimismo en la promoción y constitución de cooperativas mineras conformadas por los mineros informales. 
d. En el sector urbano actuar en la promoción y constitución de cooperativas de trabajo, de servicios educacionales, escolares, vivienda, de servicios profesionales y de otros tipos.

\section{BIBLIOGRAFÍA}

KAZTMAN, Rubén, (1996) "Notas sobre la medición de la vulnerabilidad social” (http://www.eclac.cl/deype/mecovi/docs/ TALLER5/24.pdf).

FIGUEROA ARÉVALO, Adolfo, "Exclusión social y desigualdad en el Perú" ONU Hacia el cumplimiento de los objetivos de desarrollo del compromiso del país.

Páginas web del Ministerio de Inclusión y Desarrollo Social y del Ministerio de la Producción. 